\title{
Estudio comparativo de la función cardíaca en dos poblaciones de América Latina mediante el ecocardiograma transtorácico: Atahualpa, Ecuador y Ciudad de México, México
}

\section{Comparative study of cardiac function between two populations in Latin America using the transthoracic echocardiogram: Atahualpa, Ecuador, and Mexico City, Mexico}

\author{
Juan M. García-Graullera ${ }^{1}$, Juan A. Nader-Kawachi2 ${ }^{*}$, Liliana Crespo-Serge ${ }^{1}$ y Óscar H. Del Brutto ${ }^{3}$ \\ ${ }^{1}$ Departamento de Ecocardiografía, Centro Integral de Diagnóstico y Tratamiento; ${ }^{2}$ Unidad de Neurología y Neurocirugía, Clínica de Enfermedad \\ Cerebrovascular. Hospital Médica Sur, Ciudad de México, México; ${ }^{3}$ Escuela de Medicina, Universidad Espíritu Santo, Samborondón, Ecuador
}

\begin{abstract}
Resumen
Objetivo: Comprobar las diferencias morfológicas y funcionales del corazón en dos poblaciones latinoamericanas con distintas características raciales y condiciones de vida. Métodos: Mediante el ecocardiograma transtorácico se obtuvieron datos de 206 personas: 103 del poblado de Atahualpa, Ecuador (nivel del mar, edad $\bar{x} 75 \pm 4.2$ años, 53 mujeres) y 103 habitantes de la Ciudad de México (altitud de 2,300 m, edad $\bar{x} 75 \pm 4.2$ años, 52 mujeres). Resultados: Las diferencias significativas entre Atahualpa y la Ciudad de México fueron frecuencia cardíaca, 66 vs. 80; diámetro diastólico ventricular izquierdo, 40.8 vs. 42.7; grosor del tabique, 9.8 vs. 11.6; pared posterior, 10.2 vs. 11.8; volumen-latido en centímetros cúbicos, 53.0 vs. 46.6; volumen auricular Izquierdo, 25.8 vs. 33.6; presión sistólica de la arteria pulmonar, 27.1 vs. 42.0; gasto cardíaco, 3.1 vs. 4.8; cociente E/Ea, 6.4 vs. 9.2; área mitral, 3.4 vs. 3.0. El comparativo de la función diastólica entre Atahualpa y la Ciudad de México fue: tipo 0: 2 vs. 1; tipo 1: 96 vs. 81; tipo 2: 5 vs. 20; tipo 3: 0 vs. 1. Conclusión: Las características ecocardiográficas que identifican los cambios adaptativos del corazón en Atahualpa coinciden con personas que viven a nivel del mar y con buena actividad física y en México con los habitantes de grandes altitudes y expuestos a contaminación ambiental. La función sistólica del ventrículo izquierdo fue similar en ambas poblaciones, lo que indica que los cambios adaptativos hacen posible que el corazón sea eficaz en diferentes circunstancias del ecosistema.
\end{abstract}

Palabras clave: Ecocardiograma transesofágico. Hipertensión pulmonar. Hipoxemia. América Latina. Gran altitud. Contaminación ambiental.

\begin{abstract}
Objective: To compare morphological and functional differences of the heart in two Latin American populations with different ethnicity and living conditions. Methods: Using transthoracic echocardiogram we obtained data on 206 individuals: 103 from Atahualpa, Ecuador (living at sea level, mean age: $75 \pm 4.2$ years, 53 women) and 103 inhabitants from Mexico City (living
\end{abstract}

Correspondencia:

*Juan A. Nader-Kawachi

E-mail: juan.nader.k@gmail.com

BY-NC-ND (http://creativecommons.org/licenses/by-nc-nd/4.0/).
Disponible en internet: 06-02-2020 Arch Cardiol Mex. 2020;90(3):274-283 www.archivoscardiologia.com 
at $2300 \mathrm{~m}$ above sea level, mean age: $75 \pm 4.2$ years, 52 women). Results: Significant differences between Atahualpa and Mexico were: Heart rate 66 versus 80 x', left ventricular diastolic diameter 40.8 versus 42.7, septum thickness 9.8 versus 11.6, posterior wall 10.2 versus 11.8 , stroke volume cc 53.0 versus 46.6 , left atrial volume 25.8 versus 33.6 , systolic pressure of the pulmonary artery 27.1 versus 42.0 , cardiac output 3.1 versus 4.8 , E/Ea ratio 6.4 versus 9.2, and mitral area 3.4 versus 3.0. Comparison of diastolic function between Atahualpa and Mexico was: Type 0; 2 versus 1. Type 1; 96 versus 81. Type 2; 5 versus 20 and Type 3; 0 versus 1. Conclusion: Echocardiographic characteristics that identify adaptive changes of the heart in Atahualpa are coincident with people living at sea level and with good physical activity, and Mexico City, with inhabitants living at high altitudes and exposed to environmental pollution. The systolic function of the left ventricle was similar in both populations, indicating that adaptive changes allow the heart to be effective in different circumstances of the ecosystem.

Key words: Transesophageal echocardiogram. Pulmonary hypertension. Hypoxemia. Latin America. High altitude. Environmental pollution.

\section{Introducción}

En la última década, el conocimiento sobre epidemiología de las enfermedades neurológicas no transmisibles, particularmente los padecimientos cardiovasculares y cerebrovasculares en Latinoamérica, ha aumentado gracias a los estudios epidemiológicos en diferentes zonas de la región ${ }^{1-5}$. Existe interés creciente por analizar las diferencias fisiológicas, patológicas y epidemiológicas entre distintos grupos étnicos y personas con estilos de vida diversos. En la actualidad se sabe que más de 140 millones de personas habitan en sitios por arriba de los 2,500 m de altura y muchos más se trasladan por recreación o por la constante migración de los pueblos y se ven forzados a adaptar sus organismos al nuevo hábitat ${ }^{6}$.

Se han conducido estudios que comparan y demuestran las características fisiológicas del corazón en diferentes grupos étnicos ${ }^{7}$ que tienen diversos hábitats y altitudes ${ }^{8}$.

Las características raciales y genéticas de la población de Atahualpa, así como las definiciones y la metodología del Proyecto Atahualpa, se han descrito con anteriordad ${ }^{9}$. Por otro lado, el Hospital Médica Sur se ubica en el medio urbano de la zona sur de la Ciudad de México, con un área de influencia extensa y cuyos habitantes tienen grados variados de mestizaje y viven a una altitud promedio de 2,240 $\mathrm{m}$ de altura.

El trabajo conjunto entre los investigadores del Proyecto Atahualpa y del Departamento de Neurología del Hospital Médica Sur ha permitido realizar estudios colaborativos que demuestran la variabilidad de las características fenotípicas y fisiológicas entre dos poblaciones latinoamericanas notoriamente distintas ${ }^{10}$. De acuerdo con lo anterior, este trabajo que utiliza el ecocardiograma transtorácico (ECOTT) pretende confirmar la hipótesis de que existen diferencias anatómicas y funcionales del corazón en dos poblaciones con hábitos, sitios de residencia y características raciales distintas y que además habitan en poblaciones con diferente altitud respecto del nivel del mar y diverso grado de exposición a contaminantes ambientales.

\section{Objetivo}

Éste es un estudio transversal y observacional con un diseño analítico que intenta explorar y contrastar las diferencias (contraste de hipótesis) de las variables cuantitativas y cualitativas del ECO en dos poblaciones con carga genética, hábitat y características antropométricas claramente diferentes.

\section{Sujetos y métodos}

\section{Criterios de selección}

En Ecuador se eligió al azar a 103 personas de la Parroquia de Atahualpa, que corresponde al $25 \%$ de los habitantes incluidos en la muestra total del Proyecto Atahualpa ${ }^{9}$. El único criterio de exclusión fue la voluntad expresa de no participar en el estudio. Para efectuar un análisis comparativo, en la Ciudad de México se seleccionó a 103 pacientes consecutivos de apariencia sana, que acudieron a la Clínica de Diagnóstico y Tratamiento del Hospital Médica Sur para someterse a una valoración sistemática de su estado de salud, sin que existiera enfermedad confirmada al momento de la visita. Los pacientes se seleccionaron de manera consecutiva y se parearon para igualar criterios en términos de edad y sexo con respecto a la población de Atahualpa.

\section{Descripción del hábitat y poblaciones estudiadas}

La Parroquia de Atahualpa está situada en el litoral ecuatoriano a nivel del mar y constituida desde el punto 
de vista racial por indoamericanos con escasa emigración y con un linaje relativamente común. Casi todos son trabajadores de la madera y su alimentación proviene en esencia del mar en la forma de la pesca diaria. Tanto hombres como mujeres tienen un grado de actividad física aceptable en su vida cotidiana.

La población que acude al centro integral de diagnóstico del Hospital Médica Sur procede de distintos sectores de la Ciudad de México a una altitud que fluctúa entre los 2,200 y los 2,400 m de altura. La raza de estos individuos es diversa, en su mayoría con grados variables de mestizaje entre indoamericanos del altiplano mexicano y europeos (de manera predominante de España) y del resto del mundo. La actividad laboral de estos sujetos es de tipo burocrático con poca actividad física y una alimentación diversa, incluidos los alimentos procesados.

\section{Metodología estadística}

Se utilizó la prueba de Kolmogorov-Smirnov para determinar la normalidad en la distribución de los datos. Para establecer y analizar la diferencia entre las variables continuas entre los grupos se usaron las pruebas $T$ y $Z$ con un intervalo de confianza del $95 \%$. Para sustentar la hipótesis en la que las diferencias en el ECOTT permiten describir las características entre las poblaciones se empleó la prueba de regresión logística.

\section{Metodología del ECOTT}

Los pacientes se sometieron a un examen estándar de ECOTT ${ }^{11}$. En Atahualpa se usó un ecocardiógrafo Terason Usmart 3300 portátil, Boston, MA, EE.UU., y en la Ciudad de México un ecocardiógrafo Phillips modelo IE33 y HD15, Holanda. En todos los casos se usaron los valores indexados con el fin de ajustar la variabilidad antropométrica de las dos muestras. Todos los estudios se realizaron por los mismos dos expertos (JMGG y LCS), lo que evitó así la variabilidad interoperador e interobservador.

Fue posible obtener los datos cuantitativos, cualitativos, anatómicos y funcionales del corazón por medio del modo $\mathrm{M}$, modo bidimensional, del Doppler a color, continuo, pulsado y tisular, de acuerdo con los criterios de la American Society of Echocardiography ${ }^{12}$ de la siguiente manera:

Eje largo paraesternal: medidas cuantitativas: tamaño del ventrículo izquierdo; grosor de las paredes ventriculares, diámetro de la aurícula izquierda y raíz cortical y diámetro del tracto de salida del ventrículo izquierdo.

Eje corto a nivel de ápex: medidas cualitativas: descripción de músculos papilares y de la válvula mitral, en la que se valoró su movilidad en los 17 segmentos convencionales. El resultado fue descriptivo y para fines estadístico se dicotomizó (normal contra anormal).

Eje apical de cuatro cámaras: medidas cuantitativas: se valoraron en dos dimensiones el diámetro y el volumen de ambas aurículas, y el diámetro del ventrículo derecho. Medidas cualitativas: movilidad del ventrículo izquierdo en su pared lateral y del tabique en su porción inferior.

Apical de dos cámaras: medidas cualitativas: movilidad de la pared inferior y la pared anterior del ventrículo izquierdo.

Apical de tres cámaras: medidas cualitativas: movilidad de la pared posterolateral y del tabique en su porción anterior.

Valoración de las válvulas: medidas cualitativas por medio de diferentes tomas con modo bidimensional para el análisis morfológico. Doppler a color para valorar insuficiencias valvulares, Doppler pulsado para evaluar velocidades y gradientes normales, Doppler continuo para valorar velocidades y gradientes por arriba de los límites normales. El área valvular mitral se midió por el tiempo de hemipresión.

La función diastólica se valoró con base en las guías del $2015^{11}$ con el Doppler pulsado a nivel de la vía de entrada del ventrículo izquierdo, con medición de las ondas E y A, y la relación entre ellas; y Doppler tisular para medir la onda $E$ prima lateral para la obtención de la presión telediastólica del ventrículo izquierdo, que en la mayoría de los casos es la relación del cociente de la velocidad pico de la onda $E$ mitral y la velocidad $E$ del anillo lateral mitral (E/Ea) lateral.

A partir de las medidas obtenidas se clasificó la función diastólica en las siguientes categorías: tipo $0=$ normal; tipo $=1$ relajación lenta; tipo $2=$ seudonormal; $y$ tipo $3=$ restrictivo $^{13}$. La medición de la presión pulmonar se realizó mediante el cálculo del gradiente de insuficiencia tricúspide y la suma de la presión de la aurícula izquierda (ecuación de Bernoulli) ${ }^{14}$.

La función del ventrículo izquierdo por medio de la fracción de expulsión del ventrículo izquierdo (FEVI) se determinó por aproximación de cuatro y dos cámaras en el eje apical. Se utilizó el método de Simpson biplanar. La función del ventrículo derecho se calculó por medio del modo $\mathrm{M}$ a nivel de la válvula tricúspide para 
Tabla 1. Comparación de las poblaciones: demografía

\begin{tabular}{|l|c|c|}
\hline Género & $\begin{array}{c}\text { Atahualpa } \mathbf{n = 1 0 3} \\
\mathbf{n}(\%)\end{array}$ & $\begin{array}{c}\text { Ciudad de México } \mathbf{n}=\mathbf{1 0 3} \\
\mathbf{n}(\%)\end{array}$ \\
\hline Mujeres & $53(51 \%)$ & $52(50 \%)$ \\
\hline Hombres & $50(49 \%)$ & $51(50 \%)$ \\
\hline Edad & $\begin{array}{c}\text { Promedio } \\
\text { máx./mín.-DT }\end{array}$ & $\begin{array}{c}\text { Promedio } \\
\text { máx./mín.-DT }\end{array}$ \\
\hline Todos & 75.2 & 75.2 \\
\hline Mujeres & $95 / 62-8.4$ & $94 / 59-8.4$ \\
\hline Hombres & 75.7 & 75.7 \\
\hline & $94 / 62-8.4$ & $94 / 61-8.3$ \\
\hline
\end{tabular}

detectar el movimiento de excursión sistólica del anillo tricúspide (ESAT).

El volumen latido en centímetros cúbicos (vol. lat.) se obtuvo mediante la medición de la integral de velocidad-tiempo aórtico por el área del tracto de salida del ventrículo izquierdo. El gasto cardiaco se extrajo al multiplicar este valor por la frecuencia cardíaca ${ }^{11}$.

\section{Resultados}

Se estudió a un total de 206 sujetos: 103 en Atahualpa (53 mujeres y 50 hombres), con edad promedio de $75.2 \pm 4.2$ años, y 103 en la Ciudad de México (52 mujeres y 51 hombres), con edad promedio de $74.8 \pm 4.2$ años (Tabla 1).

\section{Datos demográficos}

Características sociodemográficas: Atahualpa se localiza a nivel del mar, con actividad física de moderada a intensa de sus habitantes y etnia homogénea (amerindios) y nula contaminación. La Ciudad de México se halla a 2,300 m de altura, con actividad física de escasa a moderada y etnia diversa de sus habitantes, expuestos a diferentes contaminantes.

Antropometría: la población de México tuvo en promedio mayor estatura $(162.4 \mathrm{~cm}$ vs. $148.7 \mathrm{~cm})$, mayor peso corporal (71 kg vs. $57 \mathrm{~kg}$ ) y mayor área de superficie corporal (ASC) según el método de Dubois y Dobois $\left(1.76 \mathrm{~m}^{2}\right.$ vs. $\left.1.49 \mathrm{~m}^{2}\right), \mathrm{p}<0.05$ (Tabla 2).

Factores de riesgo cardiovascular: no se observaron diferencias en el número de personas que padecían diabetes mellitus (DM), hipertensión arterial (HTA) y dislipidemia; en cambio, el tabaquismo se registró en 12 personas de la población de México y
Tabla 2. Comparación de las poblaciones: antropometría

\begin{tabular}{|l|c|c|}
\hline Antropometría & $\begin{array}{c}\text { Atahualpa } \\
\text { Promedio } \\
\text { máx./mín.-DT }\end{array}$ & $\begin{array}{c}\text { Ciudad de México } \\
\text { Promedio } \\
\text { máx./mín.-DT }\end{array}$ \\
\hline Peso, kg & $\begin{array}{c}\mathbf{5 7} \\
84 / 32-10.2\end{array}$ & $\begin{array}{c}\mathbf{7 1} \\
187 / 157-7.4\end{array}$ \\
\hline Talla, cm & $\begin{array}{c}\mathbf{1 4 8 . 7} \\
168 / 133-9.0\end{array}$ & $\begin{array}{c}162.4 \\
187 / 140-9.6\end{array}$ \\
\hline $\begin{array}{l}\text { Área de superficie } \\
\text { corporal, m² }\end{array}$ & $\begin{array}{c}\mathbf{1 . 4 9} \\
1.86 / 1.09-0.15\end{array}$ & $\mathbf{1 . 7 6}$ \\
\hline
\end{tabular}

Tabla 3. Comparación de las poblaciones: factores de riesgo cardiovascular

\begin{tabular}{|l|c|c|}
\hline $\begin{array}{l}\text { Factores de } \\
\text { riesgo }\end{array}$ & $\begin{array}{c}\text { Atahualpa } \\
\mathbf{n}(\%)\end{array}$ & $\begin{array}{c}\text { Ciudad de México } \\
\mathbf{n}(\%)\end{array}$ \\
\hline Diabetes mellitus & $31(30)$ & $24(23)$ \\
\hline Hipertensión & $51(50)$ & $49(48)$ \\
\hline Dislipidemia & $15(15)$ & $20(19)$ \\
\hline Tabaquismo & $1(1)$ & $12(12), p<001$ \\
\hline EVC & $18(17)$ & $4(4), p<001$ \\
\hline
\end{tabular}

sólo en un caso en Atahualpa. Se identificaron antecedentes de episodios vasculares cerebrales en 18 sujetos en Atahualpa y en 4 de la Ciudad de México (Tabla 3).

\section{Variables del ECOTT}

Al comparar las poblaciones de Atahualpa y la Ciudad de México, respectivamente, se encontraron los siguientes parámetros: frecuencia cardíaca (FC), 67 vs. 80 ( $P=0.02)$; diámetro diastólico del ventrículo izquierdo (DDVI), 40.8 vs. 42.7 ( $p<0.05$ ); diámetro sistólico del ventrículo izquierdo (DSVI), 26.0 vs. 26.2; grosor del tabique IV, 9.8 vs. 11.6 ( $p<0.001)$; grosor de la pared posterior (PP), 10.2 vs. 11.8 ( $p<0.005)$; FEVI, 65.3 vs. 65.1 ; ESAT, 20.1 vs. 20.03; vol. lat., 53.0 vs. 46.6 ( $p<0.005$ ); volumen de la aurícula izquierda, 25.8 vs. 33.6 ( $p<0.005$ ); presión sistólica de la arteria pulmonar (PSAP), 27.1 vs. 42.0 ( $p<0.005)$; gasto cardíaco (GC), 3.1 vs. 4.8 ( $p<0.005)$; masa, 92.6 vs. 99.4; relación de la presión telediastólica (E/Ea), 6.4 vs. 9.2 ( $p<0.005)$; área aórtica, 2.1 vs. 2.2; área mitral, 3.4 vs. $3.0(p<0.005)$ (Tabla 4).

Función diastólica: Atahualpa: 2 individuos con función diastólica normal, 96 con disfunción diastólica tipo 1 y 5 con disfunción diastólica tipo 2; Ciudad de 
Tabla 4. Comparación de los resultados del ecocardiograma transtorácico

\begin{tabular}{|c|c|c|c|c|}
\hline Variable & $\begin{array}{c}\text { Atahualpa } \\
\text { Promedio } \\
\text { máx./mín.-DT }\end{array}$ & $\begin{array}{c}\text { Ciudad de México } \\
\text { Promedio } \\
\text { máx./mín.-DT }\end{array}$ & Valor de $\mathrm{p}$, & $\begin{array}{c}\text { Valor de } p \\
\text { sin tabaquismo }\end{array}$ \\
\hline DDVI & $\begin{array}{c}40.8 \\
58 / 27-5.4\end{array}$ & $\begin{array}{c}42.7 \\
61 / 26-4.4\end{array}$ & 0.006 & 0.005 \\
\hline DSVI & $\begin{array}{c}26.0 \\
49 / 16-5.4\end{array}$ & $\begin{array}{c}26.2 \\
47 / 15-5\end{array}$ & 0.787 & 0.691 \\
\hline Tabique & $\begin{array}{c}9.8 \\
15 / 6-1.6\end{array}$ & $\begin{array}{c}\mathbf{1 1 . 6} \\
18 / 7-1.9\end{array}$ & $<0.0001$ & $<0.0001$ \\
\hline PP & $\begin{array}{c}10.2 \\
15 / 6-1.5\end{array}$ & $\begin{array}{c}11.8 \\
35 / 7-2.9\end{array}$ & $<0.0001$ & $<0.0001$ \\
\hline FEVI & $\begin{array}{c}64.3 \\
73 / 35-7.3\end{array}$ & $\begin{array}{c}65.1 \\
81 / 25-8.8\end{array}$ & 0.503 & 0.501 \\
\hline ESAT & $\begin{array}{c}20.1 \\
29 / 14-2.9\end{array}$ & $\begin{array}{c}20.3 \\
32 / 12-3.6\end{array}$ & 0.732 & 0.775 \\
\hline Vol. lat. & $\begin{array}{c}\mathbf{5 3 . 0} \\
88.5 / 28.8-13\end{array}$ & $\begin{array}{c}46.6 \\
86 / 20.8-16.7\end{array}$ & 0.003 & 0.001 \\
\hline VAl & $\begin{array}{c}25.8 \\
73.6 / 11.1-8.6\end{array}$ & $\begin{array}{c}\mathbf{3 3 . 6} \\
95 / 12.9-12.9\end{array}$ & $<0.0001$ & $<0.0001$ \\
\hline PSAP & $\begin{array}{c}27.1 \\
42 / 18-4.8\end{array}$ & $\begin{array}{c}42.0 \\
83 / 14-10.6\end{array}$ & $<0.0001$ & $<0.0001$ \\
\hline GC & $\begin{array}{c}\mathbf{3 . 1} \\
10.2 / 1.4-1.1\end{array}$ & $\begin{array}{c}4.8 \\
9.9 / 2.2-1.4\end{array}$ & $<0.0001$ & $<0.0001$ \\
\hline Masa & $\begin{array}{c}92.6 \\
202 / 47.8-24.4\end{array}$ & $\begin{array}{c}99.4 \\
219 / 38.9-30.9\end{array}$ & 0.080 & 0.067 \\
\hline $\mathrm{E} / \mathrm{Ea}$ & $\begin{array}{c}\mathbf{6 . 4} \\
16.3 / 3.1-2.1\end{array}$ & $\begin{array}{c}9.2 \\
30.8 / 2.8-4.3\end{array}$ & $<0.0001$ & $<0.0001$ \\
\hline AA & $\begin{array}{c}2.1 \\
4.7 / 0.8-0.6\end{array}$ & $\begin{array}{c}2.2 \\
4.8 / 1-0.7\end{array}$ & 0.337 & 0.343 \\
\hline AM & $\begin{array}{c}\mathbf{3 . 4} \\
7.2 / 1.5-0.95 .4\end{array}$ & $\begin{array}{c}\mathbf{3 . 0} \\
6.7 / 1.3-1.01 .6\end{array}$ & 0.002 & 0.002 \\
\hline FC & $\begin{array}{c}\mathbf{6 7} \\
201 / 19-30.3\end{array}$ & $\begin{array}{c}80 \\
188 / 20-35.1\end{array}$ & 0.002 & 0.003 \\
\hline
\end{tabular}

AA: área aórtica; AM: área mitral; DDVI: diámetro diastólico del ventrículo izquierdo; DSVI: diámetro sistólico del ventrículo izquierdo; E/Ea: relación para la presión telediastólica; FC: frecuencia cardíaca; FEVI: fracción de expulsión del ventrículo izquierdo; GC: gasto cardíaco; masa: masa del ventrículo izquierdo, gramos por metro cuadrado; PP: grosor de la pared posterior; PSAP: presión sistólica de la arteria pulmonar; Tabique: grosor del tabique interventricular; ESAT: excursión sistólica del anillo tricuspídeo; vol. lat.: volumen latido en centímetros cúbicos; VAl: volumen de la aurícula izquierda.

México: 1 con función diastólica normal, 81 con disfunción diastólica tipo 1, 20 con disfunción diastólica tipo $2(p<0.05)$ y 1 con disfunción diastólica tipo 3 (Tabla 5).

Al considerar la diferencia en el número de personas con tabaquismo y enfermedad vascular cerebral (EVC) entre las dos poblaciones estudiadas, se realizó por separado el análisis estadístico en el cual se excluyó a las personas con dichos antecedentes y su respectivo par comparativo. En este subanálisis se advirtió que sólo la diferencia en el volumen latido (al excluir a los pacientes con EVC) fue discretamente menor, pero conservando una diferencia estadísticamente significativa ( $p=0.001$ a 0.016$)$. En ningún otro caso se modificó la diferencia en las variables del ECOTT (Tabla 5).

Al comparar a las dos poblaciones por medio del análisis interpretativo de los ecocardiogramas, en la muestra de Atahualpa se encontró a 2 pacientes con una lesión consistente con infarto del miocardio, mientras que en la Ciudad de México 7 individuos tuvieron algún daño estructural visible distribuido de la siguiente manera: infarto del miocardio (1), segmentos hipocinéticos (3), valvulopatía mitral (1), valvulopatía tricuspídea (1) e insuficiencia cardíaca congestiva (1). 


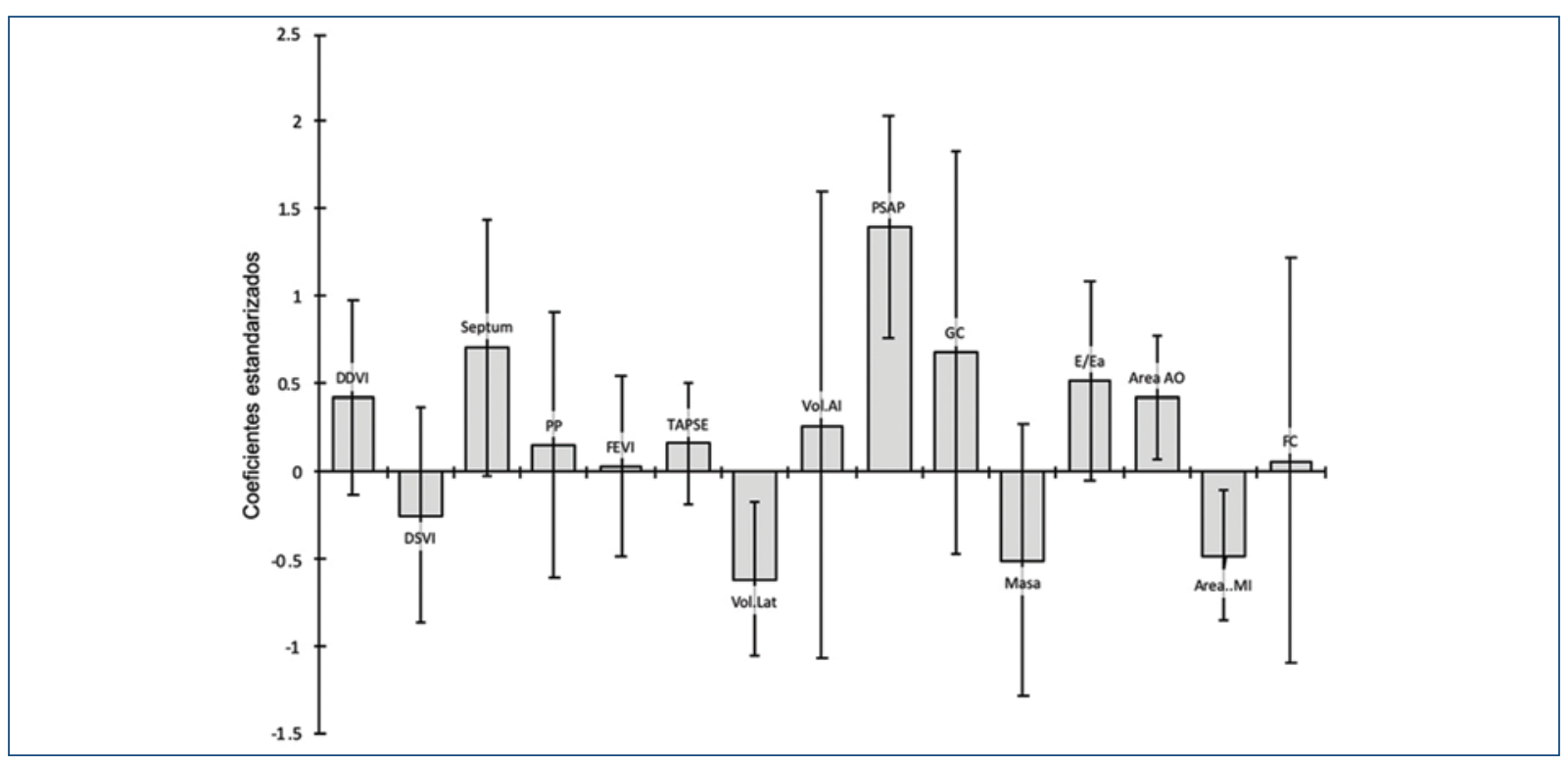

Figura 1. Coeficientes estandarizados de la regresión logística usando como variable explicativa el hábitat y las dependientes las variables del ecocardiograma (intervalo de confianza 95\%).

Área AO: area aórtica; Área MI: área Mitral; DDVI: diámetro diastólico del ventrículo izquierdo; DSVI: diámetro sistólico del ventrículo izquierdo; E/Ea: relación para la presión telediastólica; FC: frecuencia cardiaca; FEVI: fracción de expulsión del ventrículo izquierdo; GC: gasto cardiaco; Masa: masa del ventrículo izquierdo gramos por metro cuadrado; PP: grosor de la Pared posterior; PSAP: presión sistólica de la arteria pulmonar; Septum: grosor del septum interventricular; TAPSE: excursión sistólica del anillo tricúspideo; Vol. Lat.: volumen latido en centímetros cúbicos: Vol. Al: volumen de la aurícula izquierda.

Tabla 5. Tipo de función diastólica

\begin{tabular}{|l|l|c|c|}
\hline & & Atahualpa & Ciudad de México \\
\hline Tipo 0 & Normal & 2 & 1 \\
\hline Tipo 1 & Relajación lenta & $\mathbf{9 6}$ & $\begin{array}{c}\mathbf{8 1} \\
\mathrm{p}<0.05\end{array}$ \\
\hline Tipo 2 & Seudonormal & $\mathbf{5}$ & $\begin{array}{c}\mathbf{2 0} \\
\mathrm{p}<0.05\end{array}$ \\
\hline Tipo 3 & Restrictivo & 0 & 1 \\
\hline & Total & 103 & 103 \\
\hline
\end{tabular}

Mientras que el análisis comparativo permitió establecer la hipótesis según la cual existe una diferencia entre las variables del ECOTT y que ésta puede distinguir a los pobladores de uno y otro lugar, la regresión logística hizo posible confirmarla al establecer el peso de las variables. En la figura 1 se demuestran los coeficientes estandarizados resultantes de la regresión logística. En ellos se puede distinguir por medio de la dirección del signo (positivo o negativo) el comportamiento de cada una de las variables sobre la condición de vivir en Atahualpa (debajo de 0) o en la Ciudad de México (arriba de 0). De esta manera, las variables tabique, PSAP, E/ Ea y área aórtica distinguen de manera estadísticamente significativa al $90 \%$ de los habitantes de la Ciudad de México, así como las variables DDVI y GC, pero con menor poder estadístico. Por otro lado, el volumen latido y el área mitral distinguen a los pobladores de Atahualpa de forma estadísticamente significativa y la variable masa del ventrículo izquierdo, a pesar de ser distintiva de estos pobladores en el $80 \%$ de los casos, no alcanzó significancia estadística. Al considerar de manera conjunta a todas las variables mediante el análisis ROC, estas diferencias tuvieron un área bajo la curva de 0.975 . Por medio de la regresión logística, la HTA, la DM y el tabaquismo no tuvieron una relación causal que explique por sí misma los cambios en el ECOTT.

\section{Discusión}

Los seres humanos se han adaptado al medio ambiente mediante un largo proceso evolutivo que le permite su supervivencia. En las últimas décadas, la creciente urbanización de las comunidades rurales y la frecuente migración de las personas producen cambios en el estilo de vida que requieren una adaptación 
mucho más rápida y producen reacciones diversas desde el nivel molecular hasta un nivel orgánico integrativo ${ }^{15}$.

Latinoamérica es una de las regiones del mundo con mayor diversidad genética, de costumbres y hábitats. En el análisis comparativo de estos datos se confirmó la hipótesis de que existen diferencias anatómicas y fisiológicas en el corazón de los pobladores de Atahualpa y la Ciudad de México y que éstas se deben a distintos factores adaptativos.

\section{Factores étnicos y demográficos}

Las diferencias en la talla y el peso corporal, la actividad física y los factores de riesgo cardiovascular influyen sobre la función cardíaca ${ }^{16}$. Al existir una relación directa entre la talla y la masa del ventrículo izquierdo, dada la menor estatura en la población de Atahualpa, es esperable que estos individuos tengan menor masa del ventrículo izquierdo. Para homogeneizar la muestra se emplearon los valores indexados del ECO en todos los casos. En los pobladores de Atahualpa, la eficacia contráctil del ventrículo izquierdo se demuestra con un mayor volumen latido y ello permite mantener un GC efectivo aun con frecuencias cardíacas más bajas. Estos hallazgos ilustran la importancia que tiene, como sucede en las personas con mayor actividad física como los atletas de alto rendimiento ${ }^{17}$, el estilo de vida en la función ventricular; en Atahualpa, la relación entre masa del ventrículo izquierdo, volumen latido y GC describe la eficacia de la contractilidad ventricular para mantener el GC con una baja FC. Por el contrario, al ser la talla y en particular el peso corporal las determinantes condicionales del GC en los pobladores de la Ciudad de México, puede inferirse que éstas son la consecuencia de la vida urbana y sedentaria en esta población (Fig. 1).

Una diferencia interesante entre las dos poblaciones es el grosor del tabique IV. Esta diferencia no se puede atribuir a la diversidad étnica por sí misma. En un estudio de 1,818 habitantes de la comunidad latina en zonas urbanas de EE.UU. no hubo diferencias en el grosor del tabique IV ni de la PP, excepto en personas de origen cubano y caribeño, con genética africana ${ }^{7,18}$. Otros factores que influyen en el grosor del tabique son la HTA ${ }^{19}$, el tabaquismo ${ }^{20}$ y la obesidad ${ }^{16}$. La HTA, por ejemplo, produce remodelado del ventrículo izquierdo y del tabique IV ${ }^{19}$. En las poblaciones estudiadas no fue posible determinar la influencia de la HTA sobre el grosor del tabique, toda vez que su prevalencia fue similar. Contrario a lo anterior, el tabaquismo fue más prevalente en los pobladores de la Ciudad de México. Para comprender la influencia del tabaquismo en las variables del ECOTT se llevó a cabo una prueba estadística para comparar sólo a las personas sin tabaquismo en ambos grupos y se observó que, si bien las poblaciones fueron más parecidas, la diferencia aún fue estadísticamente significativa, es decir, que además del tabaquismo existen otros factores que influyen sobre el grosor del tabique. En cuanto a la obesidad, como ya se mencionó, el área de superficie corporal en la Ciudad de México fue mayor que en Atahualpa. Los cambios en el ECOTT con el sobrepeso incluyen el engrosamiento del tabique, incluso al considerar que en muchos pacientes obesos la remodelación ventricular es de tipo excéntrico y en esta serie predominó la remodelación concéntrica ${ }^{16}$.

\section{Hipoxemia y altitud}

La diferencia de la presión barométrica entre el nivel del mar y $2,500 \mathrm{~m}$ de altura reduce entre 20 y $30 \%$ la presión de $\mathrm{O}_{2}$. Esto equivale a que una persona que habita a nivel del mar se expone a una fracción inspirada de $\mathrm{O}_{2}$ del 21 al $14 \%{ }^{21}$. Dicho de otra manera, en la Ciudad de México existe 20 a 30\% menos oxígeno disponible en el aire que en Atahualpa.

Diversos estudios han investigado a las poblaciones que residen por arriba de los $3,000 \mathrm{~m}$ de altura, pero son mucho menos los que consideran a la inmensa población que habita en alturas intermedias, entre los 2,000 y 3,000 m sobre el nivel de mar. Tan sólo en la República Mexicana viven alrededor de 30 millones de personas a más de $2,000 \mathrm{~m}$ de altura y el $90 \%$ de ellos corresponde a poblaciones urbanas que han recibido migración desde zonas rurales de menor altitud ${ }^{22}$. En habitantes de gran altura, el primer mecanismo adaptativo a la hipoxia es el aumento de la $\mathrm{FC}^{23}$ y la disminución del volumen latido ${ }^{24}$. Los autores encontraron en los pobladores de la Ciudad de México mayor FC ( 80 vs. $67, p<0.002$ ) y menor volumen latido (53.0 vs. 46.6, $p<0.005)$ en comparación con Atahualpa. Estos fenómenos están mediados por el aumento de la actividad simpática. Se ha demostrado que las personas sanas que se hallan expuestas a alturas extremas tienen mayor concentración de noradrenalina en plas$\mathrm{ma}^{25}$. En un estudio reciente sobre voluntarios sanos expuestos progresivamente a hipoxia al mudarse hacia sitios de mayor altitud, se registraron valores plasmáticos elevados de noradrenalina en el momento en que migraron hacia una mayor altura y continuaron aún después de un período adaptativo de cuatro semanas $^{26}$. Se sabe además que el fenómeno inverso 
sucede cuando un habitante de altura se desplaza de un lugar elevado al nivel del mar'24.

Al ser la presión pulmonar un sistema de baja presión, pequeños aumentos en la resistencia de salida del ventrículo derecho se traducen en alteraciones fisiológicas considerables. En condiciones de altura, además de una baja concentración de $\mathrm{O}_{2}$ y una disminución del volumen de agua corporal ${ }^{8}$, aumenta el hematócrito y, debido a la vasoconstricción de las arterias pulmonares, se incrementa la resistencia en la poscarga de la circulación pulmonar ${ }^{27}$. Los pacientes que residen de manera habitual en alturas mayores de 2,000 m muestran un estado persistente de hipertensión pulmonar ${ }^{28}$. Dicho estado produce remodelación de las arterias y arteriolas pulmonares por hipertrofia e hiperplasia de la capa muscular y disminución de la luz vascular ${ }^{28,29}$. En la lógica de los procesos adaptativos de los seres vivos, la exposición a diferentes situaciones ambientales induce cambios que al final causan modificaciones de transcripción. En fecha reciente se ha descrito la vía del factor inducido por hipoxia (hypoxia-inducible factor) en pobladores de gran altitud; esta línea del conocimiento ofrece una nueva explicación que permite entender los cambios adaptativos del corazón a largo plazo ${ }^{6}$. En coincidencia con lo anterior, los datos obtenidos apuntan a la PSAP como el comparativo funcional que más distingue a las poblaciones estudiadas; la PSAP en Atahualpa fue de 27.1 y la de la Ciudad de México de 42.0, lo que revela un estado de hipertensión pulmonar persistente en esta última población. Mediante la prueba de regresión logística, la PSAP resultó ser el mayor indicador de causalidad entre la altura y las condiciones del hábitat. Relacionado con esto, la OMS incluyó en 2003 a la hipoxemia por exposición prolongada a grandes alturas como causa relacionada con hipertensión pulmonar dentro del grupo $\mathrm{III}^{30}$. La característica de este grupo es que en su mayoría son condiciones fisiológicas que pueden cambiar al modificar el factor causal.

En los pobladores de la Ciudad de México, el grosor del tabique y el de la PP del ventrículo izquierdo por remodelación concéntrica de la pared ventricular fueron mayores. A este respecto, un estudio de ECOTT en habitantes de gran altitud demuestra menos movilidad del tabique IV y más movilidad y eficiencia contráctil de la pared posterolateral del VI, lo que posibilita mantener una fracción de expulsión adecuada en condiciones de gran altura ${ }^{23}$. Por otro lado, en un estudio comparativo entre habitantes frecuentes de grandes alturas (serpas), habitantes recientemente adaptados a grandes alturas y habitantes a nivel del mar, se documentó un menor grosor del tabique IV tanto en serpas como en aquéllos adaptados a grandes alturas ${ }^{8}$. Si bien estos cambios no parecen significativos, sí son opuestos a los hallazgos presentados aquí.

Es muy interesante observar que, a pesar de las diferencias funcionales y morfológicas del corazón ya descritas, no se observaron diferencias significativas en DSVI o ESAT, lo que pone en evidencia que la normalización de la función sistólica es el mecanismo final que da lugar a la adaptación a diferentes circunstancias de altura 8 .

\section{Contaminación ambiental}

Se calcula que en el mundo 4.2 millones de muertes prematuras se deben a padecimientos relacionados con la contaminación ambiental; de éstas, $36 \%$ es efecto de enfermedad pulmonar, $34 \%$ de ictus cerebral y $27 \%$ de alguna una complicación cardíaca ${ }^{31}$. De la misma manera, la contaminación causa graves consecuencias cerebrales ${ }^{32}$, incluida la enfermedad de Alzheimer $^{33}$. Son relativamente escasos los trabajos que estudian los efectos de la contaminación ambiental sobre el corazón y, en opinión de los autores, ninguno compara a poblaciones que habiten en condiciones extremas en cuanto a la calidad del aire. En este trabajo, dicha diferencia es muy evidente; en tanto que Atahualpa existe poca o nula contaminación del aire por industria o vehículos de combustión interna, en la Ciudad de México estos factores son causas determinantes de contaminación.

En los últimos años, la concentración de $\mathrm{CO}_{2}$ ha sido relativamente contenida en la Ciudad de México; sin embargo, otros contaminantes como el dióxido de azufre, el ozono y las partículas suspendidas tipo PM10 y PM2.5 sobrepasan los valores recomendados por la OMS en el $50 \%$ de los días del año ${ }^{34}$.

En la última década se ha descrito la influencia que tiene el estrés urbano en la disfunción diastólica en jóvenes ${ }^{35}$, probablemente mediada por el péptido cerebral natriurético ${ }^{36}$, así como en mujeres mayores expuestas a la contaminación ambiental por la exposición a ozono y a partículas PM2.5 y PM10 ${ }^{37}$. En concordancia con esto, si bien es cierto que, dada la media de edad de esta población, es esperable que exista cierta disfunción diastólica en la mayoría de los sujetos, el tipo de disfunción diastólica fue más grave en la Ciudad de México. Se observó que el $96 \%$ de las personas en Atahualpa, en comparación con el $81 \%$ en la Ciudad de México, experimentaron una disfunción diastólica con patrón de relajación lenta (tipo 1), en tanto que la disfunción diastólica con patrón seudonormal (tipo 2) 
se presentó en 5 en Atahualpa y 20 en la Ciudad de México y las diferencias fueron estadísticamente significativas (Tabla 5).

Ya se ha mencionado que en los habitantes de la Ciudad de México, la masa del ventrículo izquierdo fue mayor en relación con diferentes factores. En un estudio reciente ${ }^{38}$ se demostró que la exposición constante a partículas finas PM2.5 y el dióxido de nitrógeno generan cambios fenotípicos como el aumento de la masa del ventrículo izquierdo y del volumen ventricular. Asimismo, la exposición al ruido y al tránsito producen alteraciones fenotípicas que incrementan el volumen ventricular ${ }^{38}$. En este trabajo, el DDVI fue mayor en los pobladores de la Ciudad de México, lo que indica de manera indirecta que en esta población existe un mayor volumen ventricular, relacionado entre otros factores con la exposición a contaminantes. En esta investigación no se incluyó como variable el volumen ventricular, lo que obliga a conducir un trabajo futuro sobre este punto particular.

\section{Conclusiones}

Las variables que caracterizan a los cambios adaptativos en el ECOTT en las dos poblaciones estudiadas fueron los siguientes: Atahualpa: volumen latido, masa del ventrículo izquierdo y área mitral; Ciudad de México: DDVI, tabique, PSAP, GC, relación E/Ea y área aórtica.

Los cambios adaptativos a diferentes condiciones ambientales, en especial de hipoxia, se presentan en todas las personas, cualquiera que sea el grupo étnico. Estos cambios se producen por la elevación de la presión sistólica pulmonar y la remodelación del hemicardio derecho por hipertrofia o dilatación ligera de cavidades derechas y cambios del ventrículo izquierdo menos evidentes. En este trabajo, a pesar de contar con un número limitado de individuos de estudio, la metodología comparativa permitió identificar diferencias en el ECO estadísticamente significativas entre las dos poblaciones. En este grupo, los pobladores de Atahualpa tuvieron ventrículos más pequeños y con buena movilidad, efecto de vivir a nivel del mar y de una actividad física más intensa; en cambio, los habitantes de la Ciudad de México tuvieron mayor espesor de las paredes ventriculares, dilatación auricular izquierda secundaria a la remodelación ventricular y disfunción diastólica, secundarios tanto a la altitud como al estilo de vida urbano, y muy probablemente relacionadas con la contaminación ambiental; esta última, según los postulados previos, debe por tanto considerarse como un factor de riesgo cardiovascular modificable.

\section{Puntos clave}

- El cambio de hábitat y las condiciones de vida producen cambios adaptativos en el corazón.

- Estos cambios, si bien se relacionan con la carga genética individual, tienen influencia de la altitud, la actividad física y la contaminación ambiental.

- En Latinoamérica existe un alto índice migratorio del campo hacia la ciudad.

- Este trabajo compara las características ecocardiográficas del corazón de dos poblaciones latinoamericanas genéticamente diversas y expuestas a condiciones ambientales por completo diferentes.

- Los pobladores de Atahualpa tuvieron ventrículos más pequeños y con buena movilidad, propios de los pobladores a nivel del mar y con actividad física intensa.

- Los habitantes de la Ciudad de México mostraron mayor espesor de las paredes ventriculares, dilatación auricular izquierda y disfunción diastólica, inherentes a los habitantes de sitios altos, con estilo de vida urbano y exposición a contaminantes.

- Estos cambios también describen la adaptación que necesariamente sufre el gran número de individuos que migra hacia las urbes.

- La influencia de la contaminación ambiental en el organismo humano es motivo de nuevas investigaciones, sea por este grupo o por otros grupos interesados.

\section{Conflicto de intereses}

Ninguno.

\section{Fuentes de financiamiento}

El presente trabajo fue patrocinado por la Universidad Espíritu Santo, Samborondón, Ecuador. Otros recursos fueron brindados por Servicios al Enfermo Neurológico A.C. Ciudad de México, México. Ninguno de los autores recibió remuneración económica alguna.

\section{Responsabilidades éticas}

Protección de personas y animales. Los autores declaran que para esta investigación no se han realizado experimentos en seres humanos ni en animales.

Confidencialidad de los datos. Los autores declaran que han seguido los protocolos de su centro de trabajo sobre la publicación de datos de pacientes. 
Derecho a la privacidad y consentimiento informado. Los autores han obtenido el consentimiento informado de los pacientes y/o sujetos referidos en el artículo. Este documento obra en poder del autor de correspondencia.

\section{Bibliografía}

1. Saposnik G, Del Brutto OH. Stroke in South America: a systematic review of incidence, prevalence, and stroke subtypes. Stroke. 2003;34:2103-2107.

2. Lavados PM, Sacks C, Prina L, Escobar A, Tossi C, Araya F, Feuerhake W, et al. Incidence,30-daycase-fatalityrate, and prognosis of stroke in Iquique, Chile: a 2-year community-based prospective study (PISCIS Project). Lancet. 2005;365:2206-2215.

3. Medina MT, Thompson-Cerna AR. Prevalencia de la enfermedad cerebrovascular en la comunidad rural de Salamá, Honduras, utilizando el método epidemiológico de captura-recaptura. Rev Neurol. 2007;44:460-464.

4. Cantú-Brito C, Majersik J, Sánchez B. Door-to-door capture of incident and prevalent stroke cases in Durango, Mexico: the brain attack surveillance in Durango Study. Stroke. 2011;42;3:601-606.

5. Lavados PM, Hennis AJM, Fernandes JG, Medina MT, Legetic B, Hoppe A, et al. Stroke epidemiology, prevention, and management strategies at a regional level: Latin America and the Caribbean. Lancet Neurol. 2007;6:362-372.

6. Bigham AW, Lee FS. Human high-altitude adaptation: forward genetics meets the HIF pathway. Genes Dev. 2014;28:2189-2204.

7. Qureshi WT, Leigh JA, Swett K, Dharod A, Allison MA, Cai J, et al. Comparison of echocardiographic measures in a hispanic/latino population with the 2005 and 2015 American society of echocardiography reference limits (The Echocardiographic Study of Latinos). Circ Cardiovasc Imaging. 2016;9:1-9.

8. Stembridge M, Ainslie PN, Hughes MG, Stöhr EJ, Cotter JD, Nio AQ, et al. Ventricular structure, function, and mechanics at high altitude: chronic remodeling in Sherpa vs. short-term lowlander adaptation. J App Physiol. 2014;117:334-343.

9. Del Brutto OH, Peñaherrera E, Ochoa E, Santamaría M, Zambrano M, Del Brutto VJ; Atahualpa Project Investigators. Door-to-door survey of cardiovascular health, stroke, and ischemic heart disease in rural coastal Ecuador - the Atahualpa Project: methodology and operational definitions. Int $\mathrm{J}$ Stroke. 2014;9:367-371.

10. Nader JA, Andrade ML, Espinosa V, Zambrano M, Del Brutto O. H. Technical difficulties due to poor acoustic insonation during transcranial doppler recordings in Amerindians and individuals of European origin. A comparative study. European Neurology. 2015;73:230-232.

11. Lang RM, Badano LP, Mor-Avi V, Afilalo J, Armstrong A, Ernande L, et al. Recommendations for cardiac chamber quantification by echocardiography in adults: an update from the American Society of Echocardiography and the European Association of Cardiovascular Imaging. J Am Soc Echocardiogr. 2015;28(1):1-39.e14.

12. Mitchell C, Rahko PS, Blauwet LA, Canaday B, Finstuen JA, Foster MC, et al. Guidelines for performing a comprehensive transthoracic echocardiographic examination in adults: recommendations from the American Society of Echocardiography. J Am Soc Echocardiogr. 2019;32:1-64.

13. García MJ. Diagnóstico y guía terapéutica de la insuficiencia cardiaca diastólica. Rev Esp Cardiol. 2003;56:396-406.

14. Galiè N, Humbert M, Vachiery JL, Gibbs S, Lang I, Torbicki A, et al. ESC Scientific Document Group. 2015 ESC/ERS Guidelines for the diagnosis and treatment of pulmonary hypertension: The Joint Task Force for the Diagnosis and Treatment of Pulmonary Hypertension of the European Society of Cardiology (ESC) and the European Respiratory Society (ERS): Endorsed by: Association for European Paediatric and Congenital Cardiology (AEPC), International Society for Heart and Lung Transplantation (ISHLT). Eur Heart J. 2016;37:67-119.
15. International Migration Database. OECD Statistics. https://stats.oecd.org/ Index.aspx?DataSetCode=MIG Consultado el 20 de junio de 2019.

16. Alpert MA, Terry BE, Mulekar M, Cohen MV, Massey CV, Fan TM, et al. Cardiac morphology and left ventricular function in normotensive morbidly obese patients with and without congestive heart failure, and effect of weight loss. Am J Cardiol. 1997;80:736-740.

17. Fagard R. Athlete's heart. Heart. 2003;89:1455-1461.

18. Rodríguez CJ, Dharod A, Allison MA, Shah SJ, Hurwitz B, Bangdiwala SI, et al. Rationale and design of the echocardiographic study of hispanics/ latinos (ECHO-SOL). Ethn Dis. 2015;25:180-186.

19. Aronow WS. Hypertension and left ventricular hypertrophy. Ann Transl Med. 2017;5:310.

20. Eroglu E, Aydin S, Yalniz F, Kalkan AK, Bayrak F, Degertekin M. Chronic cigarette smoking affects left and right ventricular long-axis function in healthy young subjects: a Doppler myocardial imaging study. Echocardiography. 2009;26:1019-1025.

21. Sinex JA, Chapman RF. Hypoxic training methods for improving endurance exercise performance. Journal of Sport and Health Science. 2015;4:325-332.

22. Pérez-Padilla R. Population distribution residing at different altitudes: implications for hypoxemia. Archives of Medical Research. 2002 33:162-66.

23. Hirata K, Ban T, Jinnouchi Y, Kubo S. Echocardiographic assessment of left ventricular function and wall motion at high altitude in normal subjects. Am J Cardiol. 1991;68:1692-1697.

24. Penaloza $D$, Arias-Stella J. The heart and pulmonary circulation at high altitudes. Circulation. 2007;115:1132-1146.

25. Antezana AM, Kacimi R, Le Trong JL, Marchal M, Abousahl I, Dubray C, et al. Adrenergic status of humans during prolonged exposure to the altitude of 6,542 m. J Appl Physiol. 1994;76:1055-1059.

26. Calbet JAL. Chronic hypoxia increases blood pressure and noradrenaline spillover in healthy humans. J Physiol. 2003;551:379-386.

27. Hakim TS, Michel RP, Minami H, Chang HK. Site of pulmonary hypoxic vasoconstriction studied with arterial and venous occlusion. J Appl Physiol. 2017;54:1298-1302.

28. Sylvester JT, Shimoda LA, Aaronson PI, Ward JP. Hypoxic pulmonary vasoconstriction. Physiol Rev. 2012 92:367-520

29. Hughes JMB. Hypoxic pulmonary vasoconstriction: clinical implications. Eur Respir J. 2016;47:31-34.

30. Hoeper MM. Definition, classification, and epidemiology of pulmonary arterial hypertension. Semin Respir Crit Care Med. 2009;30:369-375.

31. http://www.who.int/news-room/fact-sheets/detail/ambient-(outdoor)-air-quality-andhealth, 2018.

32. Calderón-Garcidueñas L, Calderón-Garcidueñas A, Torres-Jardón R, Ávila-Ramírez, J, Kulesza RJ, Angiulli AD. Air pollution and your brain: what do you need to know right now. Prim Health Care Res Dev. 2015:16(4):329-345.

33. Calderón-Garcidueñas L, Calderón-Garcidueñas A, Torres-Jardón R, Ávila-Ramírez J, Kulesza RJ, Angiulli AD. Brain inflammation and Alzheimer's-like pathology in individuals exposed to severe air pollution. Toxicol Pathol. 2004:32:650-658.

34. Aire Ciudad de México. Disponible en: www.aire.gob.mx http://www.aire.CIUDAD DE MÉXICO.gob.mx/descargas/publicaciones/flippingbook/informe_ anual_calidad_aire_2017/mobile/\#p=1 Consultado el 20 de junio de 2019.

35. Kapuku G, Davis H, Choksy P, Januzzi J, Harshfield G. Brain natriuretic hormone predicts stress induced alterations in diastolic function. J Card Fail. 2012;18:S11-S12.

36. Choksy P, Davis HC, Januzzi J, Thayer J, Harshfield G, Robinson VJ, Kapuku GK. Brain natriuretic hormone predicts stress-induced alterations in diastolic function. Am J Med Sci. 2014:348;366-370.

37. Ohlwein S, Klümper C, Vossoughi M, Sugiri D, Stolz S, Vierkötter A, et al. Air pollution and diastolic function in elderly women-Results from the SALIA study cohort. Int J Hyg Environ Health. 2016:219:356-363.

38. Aung N, Sanghvi MM, Zemrak F, Lee AM, Cooper JA, Paiva JM, et al. Association between ambient air pollution and cardiac morpho-functional phenotypes: insights from the UK Biobank population imaging study. Circulation. 2018:138:2175-2186. 